\title{
CEP control of multiphoton ionization using shaper- generated variable frequency ratio bichromatic fields
}

\author{
Stefanie Kerbstadt ${ }^{1, *}$, Dominik Pengel ${ }^{1}$, Tim Bayer $^{1}$, and Matthias Wollenhaupt ${ }^{1}$ \\ ${ }^{1}$ Carl von Ossietzky University, Institute of Physics, Carl-von-Ossietzky-Straße 9-11, 26129 Oldenburg
}

\begin{abstract}
We demonstrate CEP control of the directional photoemission from multiphoton ionization (MPI) of xenon atoms using $(7 \omega: 8 \omega)$ bichromatic fields whose frequency ratio is tailored to the order of the MPI process. The bichromatic fields are generated by white light supercontinuum polarization pulse shaping. In the experiment, we observe a CEP-sensitive lateral asymmetry of up to $70 \%$. The CEP sensitivity arises from interference of opposite parity continuum states coupled to the ground state by a different number of photons.
\end{abstract}

\section{Introduction}

The phase-sensitive interaction between light and matter is the key to coherent control and, for example, enables us to manipulate the directionality of quantum processes [1]. Different methods have been devised for this purpose, e.g., phase-stable few-cycle pulses [2] or $(\omega: 2 \omega)$ fields [3]. In few-cycle applications, the carrier-envelope phase (CEP) serves as control parameter, whereas in the bichromatic approach the relative phase between the colors is generally used to control quantum dynamics.
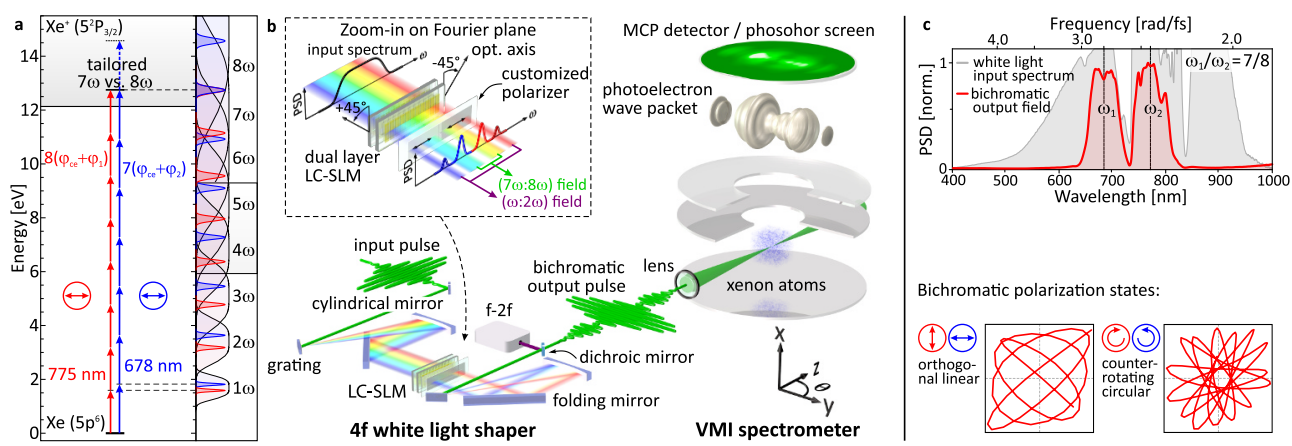

Figure 1. Excitation scheme of xenon (Xe) atoms for bichromatic 7-vs. 8-photon ionization (a) [6]. Experimental setup (b) [6]. Spectral amplitude profile of the $(7 \omega: 8 \omega)$ field (red) sculptured out of the octave-spanning WLS (gray shaded) (c) [6]. The panels below show two examples for accessible spatial polarization profiles, measured via shaper-based parametric cross-correlation trajectories $[3,5]$.

So far, $(\omega: 2 \omega)$ fields have been generated by superimposing a fundamental pulse and its second harmonic. In this case, the CEP dependence of induced multiphoton ionization (MPI)

\footnotetext{
*e-mail: stefanie.kerbstadt@uni-oldenburg.de
} 
processes vanishes [2]. In this contribution, however, we show that bichromatic fields generated by $4 f$ amplitude modulation of CEP-stable white light supercontinua (WLS) $[4,5]$ enable directional control of MPI [6] using both the CEP and the relative phase between the field components. The underlying mechanism is based on the interference of $M$ - and $N$-photon ionization pathways, with $M$ and $N$ being integer numbers of opposite parity [2]. To induce distinct $M$ - vs. $N$-photon interferences, we employ shaper-generated bichromatic fields $[1,5]$ with frequency ratios tailored to the order of the corresponding MPI process. Specifically, we apply shaper-generated $(7 \omega: 8 \omega)$ fields [6] to exert coherent control on phase-sensitive lateral asymmetries in the photoelectron momentum distribution from MPI of xenon (Xe) atoms. The shaper-based approach allows us to combine CEP-sensitive bichromatic multipath interferences with the full capabilities of femtosecond (fs) polarization pulse shaping. Shapergenerated bichromatic fields permit full optical control over individual quantum pathways and thus offer enhanced controllability of multipath interferences in the target states.

\section{Experiment}

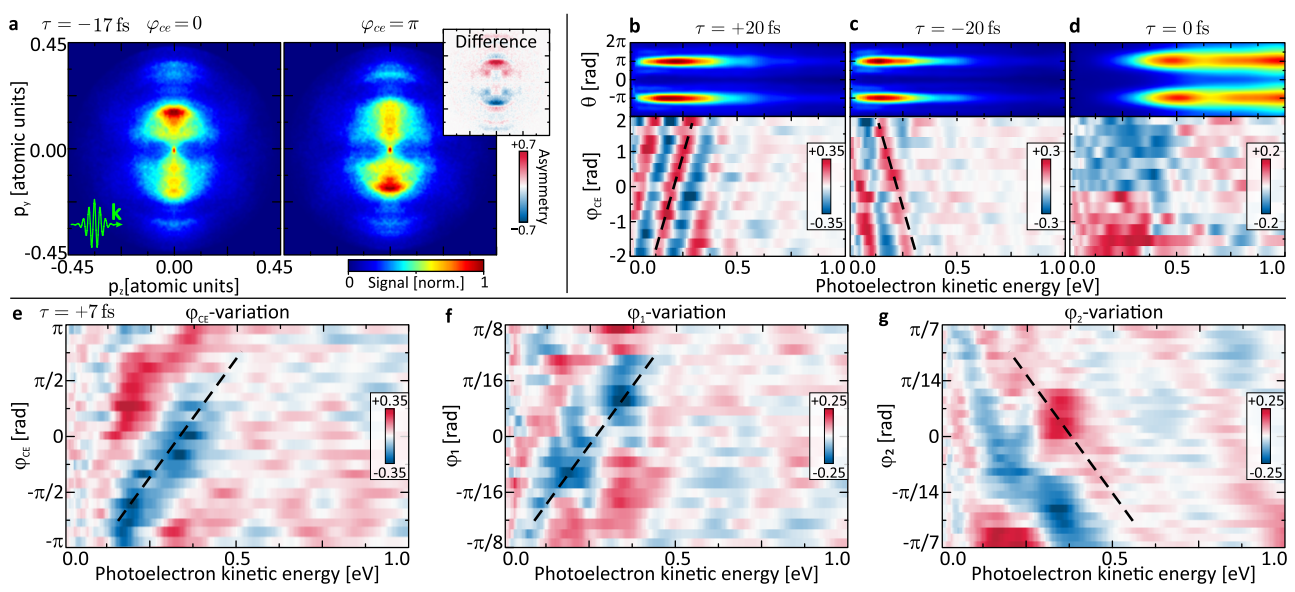

Figure 2. VMI images from time-delayed bichromatic $(7 \omega: 8 \omega)$ photoionization of xenon atoms show a pronounced CEP-sensitive lateral asymmetry up to 70\% [6] (a). Photoelectron asymmetry maps as a function of the kinetic energy and the CEP $\varphi_{c e}$ for different temporal delays $\tau$ between both colors [6] in (b)-(d). In (e)-(g): Photoelectron asymmetry maps generated by variation of the CEP $\varphi_{c e}(\mathrm{e})$, the absolute phase $\varphi_{1}$ of the low-frequency (f) and the absolute phase $\varphi_{2}$ of the high-frequency field (g).

In the experiments, we combine bichromatic pulse shaping with photoelectron imaging techniques to map asymmetries in the MPI of Xe atoms by $(7 \omega: 8 \omega)$ fields, as shown in Fig.1. Octave-spanning CEP-stable WLS from a hollow-core fiber are spectrally modulated using a $4 f$ polarization shaper equipped with a liquid crystal spatial light modulator (LC-SLM) [4-6]. By use of customized polarizers, bichromatic amplitude profiles with individually adjustable polarization state of each color are sculptured from the WLS, cf. Fig.1(b) [4, 5]. The shaperbased approach provides full access to all bichromatic parameters, such as the frequency ratio ( $M \omega: N \omega$ ), amplitude ratio, CEP $\varphi_{c e}$, relative phases $\varphi_{1,2}$ and time delay $\tau$. By application of a linear spectral phase $\varphi_{1}(\omega)=\tau \omega$, the low-frequency component is temporally delayed for $\tau<0$ and advanced for $\tau>0$. The scheme supports shaper-based dispersion management and pulse characterization [4, 5]. To investigate CEP control of bichromatic MPI of Xe, we employed p-polarized $(7 \omega: 8 \omega)$ fields with pulse duration $\Delta \tau_{1}=\Delta \tau_{2} \approx 20 \mathrm{fs}$. Active CEP stabilization was achieved by extracting a weak $(\omega: 2 \omega)$ field from the WLS, which was split off the $(7 \omega: 8 \omega)$ beam by a dichroic mirror to seed a $f-2 f$ interferometer feeding the 
CEP control loop of the laser system [6]. The $(7 \omega: 8 \omega)$ fields were focused into a velocity map imaging (VMI) spectrometer (intensity $I \approx 4 \times 10^{13} \mathrm{~W} / \mathrm{cm}^{2}$ ) for angular- and energyresolved detection of photoelectron momentum distributions, detected as a function of the CEP, as shown in Fig.2(a). The optical phases $\varphi_{c e}$ and $\varphi_{1,2}$ result in a relative quantum phase $\Delta \varphi=\varphi_{c e}+8 \varphi_{1}+\tau \omega-7 \varphi_{2}$ between the interfering electronic wave functions, determining the photoelectron asymmetry. A pronounced asymmetry up to $70 \%$ in the photoelectron momentum distribution is observed along the laser polarization direction and inverted by switching $\varphi_{c e}=0$ (left panel) to $\pi$ (right panel). The CEP dependence of the lateral asymmetry is analyzed in Figs.2(b)-(d) by means of energy-resolved asymmetry maps [6]. In case of time-

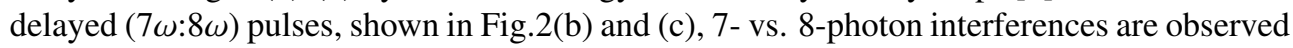
around $0.2 \mathrm{eV}$. The temporal separation of the two colors results in a linear phase between the interfering bands, leading to an energy-dependent offset of the interference pattern in $\varphi_{c e}$-direction. Consequently, the CEP interference pattern tilts, analogous to the case of linearly chirped few-cycle excitation [7]. Reversal of the pulse ordering inverts the sign of the tilt. For zero-time delay, shown in Fig.2(d), the interference pattern is aligned parallel to the $\varphi_{c e}$-axis. In this case, additional contributions from high-order frequency mixing $[6,8]$ arise in the photoelectron spectrum (top frame) at electron energies $>0.5 \mathrm{eV}$. To compare coherent control of bichromatic MPI via the CEP and the relative phase between both colors, the asymmetry maps depicted in Figs.2(e)-(g) were generated under variation of $\varphi_{c e}, \varphi_{1}$ and $\varphi_{2}$, respectively. Due to the time delay $\tau=+7 \mathrm{fs}$, the interference patterns are tilted again. In (e), $\varphi_{c e}$ was varied from $-\pi$ to $\pi$, the asymmetry map thus undergoes a full inversion cycle of the interference pattern. Although the $\varphi_{1}$-asymmetry map in (f) ranges only from $\varphi_{1}=-\pi / 8$ to $\pi / 8$ a similar asymmetry inversion cycle is covered. The results show that bichromatic MPI is much more sensitive to $\varphi_{1}$ as compared to $\varphi_{c e}$, because the $\varphi_{1}$-contributions to $\Delta \varphi$ are weighted with a factor of 8 . Analogously, the sensitivity of MPI to $\varphi_{2}$ is weighted with a factor of 7 . Therefore, $\varphi_{2}$ was varied from $-\pi / 7$ to $\pi / 7$ to achieve an equivalent interference pattern as in (e) and (f). The slope of the asymmetry map in (g) is inverted due to the negative contribution of $\varphi_{2}$ to $\Delta \varphi$.

\section{Conclusion and outlook}

In this paper, we used a novel shaping scheme to generate CEP-stable bichromatic fs pulses $[4,5]$. Tailored $(7 \omega: 8 \omega)$ fields were applied to control directional photoemission from MPI of Xe atoms along the laser polarization. The mechanism was discussed in terms of CEPsensitive 7 - vs. 8-photon pathway interferences in the $5 P_{3 / 2}$ continuum, addressing opposite parity states. The shaping scheme opens new perspectives to a broad range of applications, including, high harmonic generation and two-color pump-probe experiments [8].

\section{References}

[1] M. Shapiro, P. Brumer, "Quantum Control of Molecular Processes”, 2nd ed. (Wiley, 2012) p. 562.

[2] M. Abel, D. M. Neumark et al., Laser. Phot. Rev. 5, 352 (2011).

[3] D. Miloševic, W. Becker et al., Phys Rev. A 61, 063403 (2000).

[4] S. Kerbstadt, L. Englert, et al., J. Mod. Opt. 64, 1010 (2017).

[5] S. Kerbstadt, D. Timmer, et al., Opt. Express 25, 12518 (2017).

[6] S. Kerbstadt, D. Pengel, et. al., Phys. Rev. A 97(6), 063402 (2018).

[7] T. Pfeifer, M. J. Abel, et al., Opt. Lett. 34, 1819 (2009) .

[8] S. Kerbstadt, D. Pengel, et al., New J. Phys. 19, 103017 (2017). 